\title{
Effektives Lehren und Lernen in der Informatik, Wirtschaftsinformatik und verwandten Fachgebieten
}

\author{
Mathias Ellmann ${ }^{1}$ \\ Angenommen: 29. November 2021 / Online publiziert: 17. Februar 2022 \\ (c) Der/die Autor(en) 2022
}

\section{Zusammenfassung}

In diesem Artikel behandeln wir unser Lehrbaummodell mit den Lehr- und Lernmethoden, welches zum effektiven Lehren und Lernen in der Informatik, Wirtschaftsinformatik und verwandten Fachgebieten hinführt. Das Lehrbaummodell hat in seinen Stamm Planung und Motivation als wichtigste Lehrmethodiken auf dem effektives Lehren und Lernen basiert. Die Blätter des Lehrbaummodells wie Kommunikation, Material, Gedächtnis, Aufmerksamkeit, Lernstörung und Aktivierung im Unterricht stellen notwendige Teile des effektiven Lehren und Lernens dar und schließlich folgt der Gipfel: die Überprüfung auf Effektivität des Unterrichts und der Lehrmethoden. Wir zeigen Wege auf, wie der Wandel von traditionellen Lehrmethoden wie des Haltens eines Vortrags oder einer Vorlesung mit wenig Interaktion zu moderneren, effektiveren Methoden durch die Verwendung des Lehrbaummodells stattfinden kann. Der Artikel zeigt auf, wie Frustration im Lehren und Lernen in der Informatik, Wirtschaftsinformatik und verwandten Fachgebieten vermieden und effektiver gelehrt und gelernt werden kann. Wir versuchen, mit diesen Artikel einen Wandel von traditionellen Lehrmethoden zu psychologisch moderneren Lehrmethoden zu ermöglichen.

\section{Einleitung und Problemstellung}

Zufriedenheit bei Lernenden kann in der Informatik oder Wirtschaftsinformatik in der Lehre zur Erreichung von mehr Lehr- und Lernzielen führen [13, 14]. Im Gegensatz dazu können, sofern Erwartungen, Bedürfnisse und Motive der Lernenden an den Kurs, die Inhalte, den Lehrenden und die Lehre nicht erfüllt werden, Frustration entstehen [9, S. 36] [22, S. 19]. Frustration kann zur Aggression führen, welche im Unterricht von den Lernenden aufgrund auferlegter Tabus (sofern durch Feedbackgespräche nicht anders eingerichtet) nicht geäußert werden kann [9, S. 22-26], womit die Aggression verdrängt wird und die geistige Kapazität der Lernenden dadurch gemindert werden könnte [9, S. 36], sodass das Erreichen von Lehr- und Lernzielen gefährdet wird und ineffektiv ist.

Die entstandene Frustration bei den Lernenden kann die Erreichung der Lehr- und Lernziele stark beeinträchtigen. Sofern Lehrende die Lernenden weiterhin frustrieren, kann dies zu Dauerfrustration und neurotischen Tendenzen sowie

Mathias Ellmann

mathias.ellmann.cs@gmail.com

1 DIPLOMA Private Hochschulgesellschaft mbH, Bad Sooden-Allendorf, Deutschland psychosomatischen Symptomen führen (sofern die Lernenden die entstandene Frustrationsquelle nicht beseitigen oder tolerieren [9, S. 35]). Frustrationsquellen in der Lehre können sich nicht nur auf die Lehrenden und deren Lehre, sondern auch auf die Materialien des Lehrenden beziehen. Die entstandene Frustration bei den Lernenden kann zu Psychospielen führen [21, S. 27-28], Lernende können zu Verfolgern und Lehrende zu ihren Opfern werden. Schwache Lehrende kann dies zermürben, ihnen Energie rauben (welche sie eigentlich für die Gestaltung ihrer Lehre bräuchten) und ungesund sein [21, S. 4].

Aktivierende Lehr- und Lernmethoden können die Stunden der Nacharbeit des Unterrichts reduzieren und die Lernenden entlasten, was sowohl im Umschulungsbereich als auch in der Fernlehre und im Fernlernen [13] für Studenten, welche neben dem Beruf studieren, von Vorteil ist. Eine effektive Gestaltung der Lehre kommt Lernenden im Umschulungsbereich zugute [14]. Diese haben meist Familien, welche Aufmerksamkeit benötigen, womit eine Gefahr für das Bestehen einer IHK-Fachinformatikerprüfung allgegenwärtig ist und die Investition für eine Umschulung durch den Bildungsgutschein von Seiten des Staates durch effektive Lehre und effektives Lernen nicht fehlschlägt.

Der Kulturwandel von autoritärem zu gemeinsamem Lehren und Lernen muss geschehen, um so effektivere Lehre zu gestalten. Traditionelle Lehrmodelle müssen 
aufgegeben und psychologisch sinnvollere Lehrmodelle müssen in der Lehre in der Informatik, Wirtschaftsinformatik und verwandten Fachgebieten angebracht werden. Dieser Artikel stellt unser Lehrbaummodell vor, welches Aspekte zu einer psychologisch sinnvolleren Lehre umfasst und beschreibt.

\section{Lehrbaummodell für effektive Lehre}

Im Folgenden behandeln wir die Aspekte des Lehr- und Lernmodells (siehe Abb. 1), das Lehrbaummodell. Das Lehrbaummodell ähnelt einem Baum, bei dem der Stamm und die benannten Lehr- und Lernaspekte die stärkste Gewichtung haben. Eine Planung des Unterrichts ist notwendig, da ansonsten der Lernende keine Sicherheit und Orientierung im Unterricht erfährt, das den Lernenden frustriert und seine Lernleistung mindert [9, S. 36]. Ein Mangel an Motivation kann Lernstörungen bewirken (nach Corell [9, S. 188-202]), was den Lehr- und Lernerfolg insgesamt gefährdet. Die Überprüfung des Unterrichts auf Effektivität am Baumgipfel zeigt bspw. Schwachstellen in der Konzeption des Unterrichts auf. Die restlichen Teile

\section{Überprüfung}

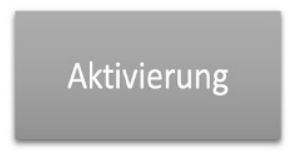

Lernstörung
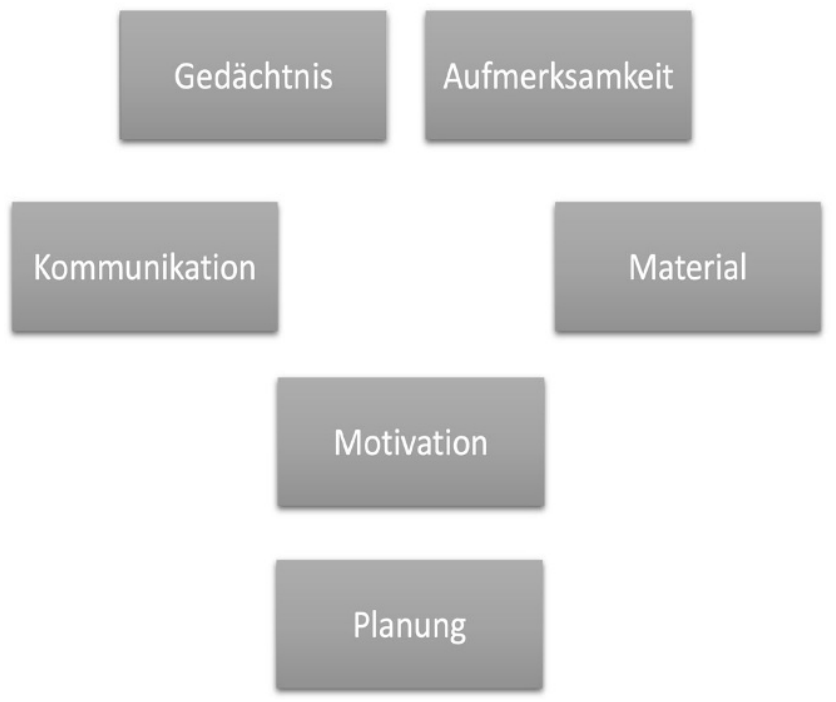

Abb. 1 Lehrbaummodell für effektives Lehren und Lernen in der Informatik, Wirtschaftsinformatik und verwandten Fachgebieten nach Ellmann und Aspekte des Lehrbaummodells sind weitere, notwendige Aspekte zur effektiven Gestaltung der Lehre, welche im Folgenden diskutiert werden. Das Lehrbaummodell hat keinen Anspruch auf Vollständigkeit und kann beliebig mit weiteren Lehraspekten erweitert werden, welche zum effektiven Lehren und Lernen beitragen.

\section{Lehren und Lernen durch Planung des Unterrichts}

Die Lehr- und Lernplanung und daraus resultierende Definition von Lehr- und Lernzielen ermöglicht nach Lew Wygotski eine notwendige Leitung [7, S. 270][26, S. 24-25] und Orientierung im Unterricht. Sofern man Lernzieltaxonomien vergleicht [4], so erscheint die Taxonomie-Matrix von TAMAS zur Analyse und Selbstevaluation von Hochschullehre [16] praktikabel, um die Lernziele für den Unterricht zu planen. Die Taxonomie-Matrix von TAMAS schlüsselt sich in die Wissensdimension (faktisch, konzeptionell, prozedural, metakongnitiv) und die kognitive Prozessdimension (erinnern, verstehen, anwenden, analysieren, beurteilen, [er]schaffen) auf und ermöglicht bei der Planung der Lehre die notwendige Sicherheit, womit die Effektivität der Lehre durch zeitliche und inhaltliche Planung der begrenzten Lehr- und Lernzeit sichergestellt ist. Sicherheit ist als Grundmotiv neben Geborgenheit in Deutschland im Laufe der Jahre gestiegen [9, S. 54]) und stellt somit das häufigste Grundmotiv bei den Lernenden dar, das in der Lehre befriedigt werden muss, sodass Frustrationen vermieden werden und die Lehre effektiv gestaltet ist.

Beispielhaft beschreiben wir die Anwendung von TAMAS [16] (siehe Abb. 2) für einen Kurs für das Design der Entwicklung einer Datenbank (siehe [14]). In einem Kurs für das Design und die Entwicklung einer Datenbank kann Faktenwissen die Speicherung von Daten in einer Tabelle sein. Es obliegt dem konzeptionellen Wissen, dass Tabellen von einem Datenbankmanagementsystem verwaltet werden. Das prozedurale Wissen ist Wissen, das notwendig ist, um Tabellen in einer Datenbank zu erstellen. Das metakognitive Wissen entsteht, wenn das faktische, konzeptionelle und prozedurale Wissen bspw. innerhalb eines individuellen Projekts zum Design und der Entwicklung einer Datenbank angewandt werden (es entsteht Wissen zum faktischen, konzeptionellen und prozeduralen Wissen).

Es soll sich an das relevante Wissen erinnert werden können (bspw. nachdem es ein Lehrender während eines praktischen Projektes gezeigt hat). Das Wissen zum Datenbankdesign kann durch eine praktische Anwendung eine praktische Bedeutung zugeordnet bekommen und dadurch verstanden werden. Die Lernenden wenden das Wissen an ihrem eigenen Projekt an und analysieren eventuelle Unterschiede zum Dozentenprojekt. Die Lernenden beurteilen die eigene Lösung und die Lösung anderer. Die Lernen- 


\begin{tabular}{|c|c|c|c|c|c|c|c|c|c|c|c|}
\hline \multirow{2}{*}{$\begin{array}{l}\text { Knowledge } \\
\text { Dimension } \\
\text { (Wissens. } \\
\text { dimension) }\end{array}$} & \multicolumn{11}{|c|}{$\begin{array}{l}\text { Cognitive Process Dimension } \\
\text { (Kognibve Prozessdimension) }\end{array}$} \\
\hline & 1 & $\begin{array}{c}\text { Remember } \\
\text { (erinnern) }\end{array}$ & 2 & $\begin{array}{l}\text { Understand } \\
\text { (verstehen) }\end{array}$ & 3 & $\begin{array}{c}\text { Apply } \\
\text { (anwenden) }\end{array}$ & 4 & $\begin{array}{c}\text { Analyse } \\
\text { (analysieren) }\end{array}$ & 5 & $\begin{array}{c}\text { Evaluate } \\
\text { (beurteilen) }\end{array}$ & $6 \begin{array}{c}\text { Create } \\
\text { ([er-]schaffen) }\end{array}$ \\
\hline \multicolumn{12}{|l|}{$\begin{array}{c}\text { A } \\
\text { Factual } \\
\text { (faktisch) }\end{array}$} \\
\hline \multicolumn{12}{|l|}{$\begin{array}{c}\text { B } \\
\text { Conceptual } \\
\text { (konzeptionell) }\end{array}$} \\
\hline \multicolumn{12}{|l|}{$\begin{array}{c}\text { C } \\
\text { Procedural } \\
\text { (prozedural) }\end{array}$} \\
\hline $\begin{array}{c}\text { D } \\
\text { Metacognitive } \\
\text { (metakognitiv) }\end{array}$ & & & & & & & & & & & \\
\hline
\end{tabular}

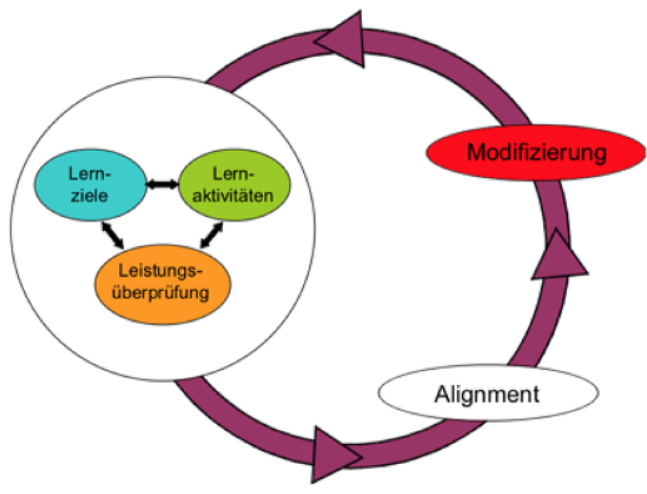

Abb. 2 Lehr- und Lernplanung nach Anderson und Krathwohl [2] und TAMAS [16] (Quelle der Abbildung)

den erschaffen eigene Lösungen am Beispiel eines Dozentenprojektes. Das Erreichen einzelner Lernziele während dieses Lernprozesses ermöglicht die Zusammensetzung der Lernergebnisse zu einem in sich logisch geschlossenen gesamten Lerngegenstand.

Nach Abb. 2 und TAMAS [16] sollte die Lehre im Hinblick auf eine Übereinstimmung der erreichten Lernziele und Lernergebnisse modifiziert werden. Es findet ein sogenanntes Alignment statt. Die Lernziele können durch Lernaktivitäten wie zum Beispiel in einem Praxisprojekt zur Datenbankentwicklung erreicht werden und überprüft werden.

\section{Lehren und Lernen durch Motivation im Unterricht}

Wirksames und effektives Lehren und Lernen kann durch Motivation geschaffen werden [9, S. 158-162]. Durch eine zielgerechte sekundäre Motivation [9, S. 67-70], wie verbale oder materielle Anerkennung und Lob [9, S. 159], sind weniger Wiederholungen und Überprüfungen notwendig [9, S. 158], um den Lerngegenstand sicher zu erfassen, was die Effektivität des Lernens und auch des Lehrens steigert. Der Lernende kann durch Selbsterfahrung [9, S. 160] oder Selbstwirksamkeit [14] unabhängig von äußeren Bestätigungen, wie bspw. Formen des Lobs und der Anerkennung, sekundärer Motivation werden, womit der Lernende primär motiviert [9, S. 67-70], sinnerfüllt [9, S. 70-73][18, S. 133] und effektiv dem Lernen nachgeht. Dies kann beispielsweise in der Informatik, Wirtschaftsinformatik und verwandten Fachgebieten durch die Umstellung der Lehre von Frontalunterricht auf ein Praxisprojekt ermöglicht werden, in dem Lernende selbst tätig werden [14] und ihr eigenes Projekt bewältigen.

Um den Lernenden zu motivieren, können verschiedene Motivierungtechniken angewandt werden [9, S. 89-91]:

- Motivierung durch Sprache [9, S. 91-97]:
Corell spricht davon, dass Menschen mit einem der fünf Grundmotive angesprochen werden sollten (siehe Tab. 1), sodass die Lernenden aktiviert werden, effektiv ihrer Tätigkeit wie dem Lernen nachzugehen. Wie in Tab. 1 aufgeführt, unterscheidet Correll fünf Grundmotive, welche in seiner Veröffentlichung in einem bestimmten Maß in Deutschland auftreten [9, S. 47-66]. Die Grundmotive wechseln sich ab, und eines steht immer an erster Stelle zur Befriedigung [9, S. 66].

Ein Lernender, der beispielsweise nach sozialer Anerkennung strebt, kann durch bspw. das Material, welches darauf abzielt, nachdem Erlernen desselben ein höheres $\mathrm{Ma}$ an sozialer Anerkennung zu erfahren, leichter durch geeignete Ansprache gelehrt und vermittelt werden. Zum Beispiel kann im Studiengang Wirtschaftsinformatik und IT-Management eine Position im Management erlangt werden, wenn der Lerngegenstand durchdrungen und verstanden wurde. Für einen Abschluss in Entrepreneurship und die Gründung einer IT-Hauses [24] lassen sich demnach nur wenige motivieren, da in Deutschland (zumindest 1996) nur 2\% der Menschen zumeist nach Unabhängigkeit und Verantwortung streben.

- Motivierung durch Bilder [9, S. 97-100]:

Die Motivierung durch Bilder kann auch dadurch erfolgen, dass sie die Motive des Lernenden anspricht. Zum

Tab. 1 Grundmotive des Menschen nach Correll [9, S. 47-66] mit ihren Bestrebungen

\begin{tabular}{ll}
\hline Grundmotiv & $\begin{array}{l}\text { Vertreten in Deutsch- } \\
\text { land (1996) }\end{array}$ \\
\hline $\begin{array}{l}\text { Soziale Anerkennung (Prestige, Überlegen- } \\
\text { heit, Status, Geltung) }\end{array}$ & $14 \%$ \\
$\begin{array}{l}\text { Sicherheit und Geborgenheit (Unauffällig- } \\
\text { keit, Gesundheit, Sparsamkeit) }\end{array}$ & $38 \%$ \\
$\begin{array}{l}\text { Vertrauen (Nähe zur Bezugspersonen) } \\
\text { Selbstachtung (Übereinstimmung Normen \& }\end{array}$ & $10 \%$ \\
$\begin{array}{l}\text { Werte) } \\
\text { Unabhängigkeit und Verantwortung (Selbst- } \\
\text { ständigkeit, Eigenverantwortung) }\end{array}$ & $2 \%$ \\
\hline
\end{tabular}


Beispiel kann eine Vorlesung zur Informatik mit Bildern, welche auf gute Job-Chancen hinweisen, das Motiv der Sicherheit und Geborgenheit ansprechen. Den Lernenden, die nach Prestige, Überlegenheit oder Status streben, kann das Aufzeigen einer Zukunft als Manager oder in einer Leitungsposition helfen, motiviert den Lerngegenstand zu erlernen.

Neben Werner Correll [9] meint David C. McClelland [7, S. 322-323], dass durch das Streben nach Befriedigung der Hauptbedürfnisse nach Macht, Erfolg oder Leistung und Zugehörigkeit der Mensch größtenteils unbewusst der Arbeit und schließlich effektiv dem Lehren und Lernen nachgeht.

\section{Lehren und Lernen durch Aktivierung im Unterricht}

Nach der Lernpyramide kann mittels Üben durch Tun und dem Unterrichten anderer mehr an Wissen, Lehr- und Lernzielen vermittelt werden [19, S. 67][18, S. 172][13, 14]. Üben durch Tun lässt neuronale Leitungsbahnen entstehen, sodass Handlungen unbewusst, automatisch ausgeführt werden können [8, S. 100-101]. Sofern die Lernenden aktiv am Lernprozess teilnehmen können, wie in einen Praxisprojekt, in dem Lehrinhalte vermittelt werden [14], so kann der Lernende die notwendigen Handlungen mit seinen Fähigkeiten zur Lösung eines Programmierproblems automatisch und effektiv anwenden. Bis zu automatischem Handeln oder unbewusster Kompetenz werden vier Kompetenzarten erlangt, nämlich unbewusste Inkompetenz, bewusste Inkompetenz, bewusste Kompetenz und unbewusste Kompetenz [8, S. 101]. Die effektivste Lernmethode ist Lernen durch Einsicht, da die Anzahl der Wiederholungen zur Erreichung einer Perfektion oder das Beherrschen einer Kette Handlungen erheblich verringert wird [9, S. 147], was wir im Rahmen eines Praxisprojektes in der Lehre bestätigen können [14]. Bis zum Lernerfolg bedarf es dennoch Übung und Anstrengung [18, S. 172].

Jerome Bruner bestätigte in seiner Forschung, dass das Lehren bedeutet, den Lernenden aktiv am Lernprozess teilhaben $\mathrm{zu}$ lassen, um Wissen $\mathrm{zu}$ erwerben [7, S. 164-165][26, S. 24-25]. Auch Werner Corell stellte fest, dass aktives Lernen passiven Lernen überlegen ist [9, S. 168-172], da dadurch der Lerngegenstand nach der Vermittlung reproduziert und beherrscht wird [9, S. 168-169] im Gegensatz zum passiven Lernen wie durch einen Vortrag. Die Teilnahme am Lernprozess kann dadurch erfolgen, dass das Wissen zur Erreichung der Lernziele in einem Projekt mit Begleitung eines Lehrenden vermittelt wird und der Lernende das Wissen im Projekt anwendet [14]. Das aktive Lernen bedeutet damit effektiver zu lernen.

Zuletzt sei erwähnt, dass eine Transferleistung nur eingeschränkt und bedingt beim Lernenden möglich ist [9,
S. 102]. Ein Lernender wird bei einer neuen Problemstellung identische Elemente oder Lernsituationen vorangegangener Problemstellungen und Lernsituationen wieder erkennen [9, S. 135]. Eine Vielzahl von Lernsituationen, in denen einen Vielzahl von Elementen gelernt wird, kann innerhalb eines Praxisprojekts, bei dem ein Lernender selbst aktiv ist, ermöglicht werden [14]. Eine Vielzahl von bereits bekannten Lernsituationen ermöglicht effektives und nachhaltiges Lehren und Lernen, da dadurch sichergestellt ist, dass eine Vielzahl von identischen Elementen in zukünftigen Problemstellungen bereits gesehen wurde und diese effektiver gelöst werden können (siehe auch [14]).

\section{Lehren und Lernen durch Material im Unterricht}

Die Lernpyramide des US National Training Laboratories [19, S. 67][18, S. 172] unterscheidet nach sieben Lehrmethoden, um bspw. das Lehrmaterial bereitzustellen. Je höher die Effektivität ist, desto wahrscheinlicher ist es, dass das Wissen bereits vor Ort effektiv vermittelt und vom Lernenden aufgenommen werden kann. Dies verringert etwaige Nacharbeit des Gelernten.

Lehr- und Lernmethode: Vortrag (Effektivität: $5 \%$ ). Es können Folien aufbereitet werden. Diese Folien sollen Sachverhalte veranschaulichen, denn Anschauliches ist leichter zu lernen als Abstraktes [9, S. 173-174], womit ein effektiverer Unterricht gestaltet werden kann.

Lehr- und Lernmethode: Lesen (Effektivität: 10\%). Es können Studienhefte geschrieben und geführt werden. Hierbei sollte darauf geachtet werden, dass Lehrziele definiert und Übungen zur Selbstkontrolle angeboten werden [13].

Lehr- und Lernmethode: Audio-Visuell (Effektivität: 20 \%). Es können Videos zur Informatik [14, 15] erstellt oder herangezogen werden. Hierbei sollte auch wieder Konkretes Abstraktem vorgezogen werden und der Unterricht ergänzt werden [14].

Lehr- und Lernmethode: Demonstration (Effektivität: 30 \% ). Der Lehrende demonstriert den Lerngegenstand. Der Lernende sollte in die Demonstration miteinbezogen und aktiviert werden, um so effektiver $\mathrm{zu}$ lehren und $\mathrm{zu}$ lernen (siehe Beispiel unter [14]).

Lehr- und Lernmethode: Diskussionsgruppen (Effektivität: $50 \%$ ). Diskussionsgruppen können an einem Lerngegenstand arbeiten und die Ergebnisse gemeinsam aufbereiten und vortragen. Die Gruppen sollten jedoch nicht $\mathrm{zu}$ groß sein, um soziales Faulenzen [18, S. 241] zu vermeiden, das ineffektiv ist.

Lehr- und Lernmethode: Üben durch Tun (Effektivität: $75 \%$ ). Dem Lernenden sollte genügend Raum geboten werden, die Inhalte zum Lerngegenstand selbst auszuüben. Beispielsweise kann ein Praxisprojekt, in dem die Lernenden selbst tätig werden, angeboten werden [14]. 
Lehr- und Lernmethode: Andere unterrichten (Effektivität: $90 \%$ ). Die Lehrenden können kleine Gruppen bilden, in denen die Lernenden jeweils die anderen Lernenden unterrichten und Materialien zur Verfügung stellen.

Lehrinhalte sollten nach George Armitage Miller [7, S. 170-173] in sinnvollen Einheiten, sogenannten Chunks, angeboten werden. Auch Werner Corell spricht von kleinen Teilschritten, in denen ein Lernender aktiv sein sollte [9, S. 169], um den Lerngegenstand schlussendlich zu beherrschen. Die Aufbereitung der Materialien in sinnvollen Einheiten und Vermittlung in kleinen Teilschritten erhöht die Effektivität des Lehrens und Lernens.

Sicherlich ist das Gesagte zur Aufmerksamkeit auch auf die Aufbereitung von Materialien übertragbar. Materialien, welche nach objektiven Aspekten (Bewegung und Veränderung, Neuheit und Einmaligkeit, Größe, Intensivität des Reizes, Dauer bzw. Wiederholungen, Faktor der Farbe) oder nach Zweck bzw. subjektiven Aspekten (Zweck oder Einstellung des Augenblicks, Pflichtbewusstsein, Erziehung, Gewöhnung, Anküpfung an Triebtendenzen) aufbereitet sind, mögen effektiver sein [9, S. 150-158]. Zudem hat das Gesagte zur Motivation auch hier Gültigkeit. Die Motivierung durch Bilder und Wörter, welche auf die Grundmotive des Menschen abzielen sollten, kann die Effektivität von Materialien und des vermittelten Wissens erhöhen.

\section{Lehren und Lernen durch Kommunikation im Unterricht}

Eine aktive Teilnahme am Lernprozess bei einem Projekt, welches der Lehrende anleitet, bedarf mehr Kommunikation zwischen den Lernenden und Lehrenden als bei einem Vortrag. Kommunikation ist nach Christel Petitcollin aufgrund von Psychospielen in der Beziehung LehrenderLernender, welche durch ineffektive Kommunikation ausgelöst werden, eine komplexe Angelegenheit [21, S. 55]. Eine effektive, gesunde und klare Kommunikation muss nach Christel Petitcollin auf Augenhöhe [21, S. 56] oder nach Patrice Ras verantwortlich und ebenbürtig stattfinden [22, S. 45], sodass eine gesunde Beziehung zwischen Lehrenden und Lernenden geschaffen wird. Dies ermöglicht die gemeinsame Gestaltung des Unterrichts und der Teilnahme am Lernprozess. Im Gegensatz dazu kann eine autoritäre Erziehungsform eine Ursache für Lernstörungen sein und die Effektivität des Unterrichts gefährden [9, S. 202-206]. Auch Robert Levine meint [20, S. 346], dass gute Lehrende darauf hinarbeiten sollen, Autoritäten in Frage zu stellen (den Lehrenden eingeschlossen). Für uns ist deshalb der Lehrende eher ein Lernbegleiter, welcher die Regeln und Inhalte vermittelt, die bei der Gestaltung einer Anwendung in der Informatik und Wirtschaftsinformatik Anwendung finden. Ein Lehrender bildet, wie Robert Levin es sagt [20,
S. 346], zum selbstständigen und kritischen Denken aus. Der Lehrende schafft eine Kultur und ein Klima des Respekts füreinander und bezüglich abweichender Meinungen [20, S. 346][9, S. 434].

Eine gesunde Kommunikation und Selbstbehauptung besteht nach Patrice Ras darin, ehrlich seine Wünsche, Gefühle und Meinungen zu äußern, ohne den anderen anzugreifen [22, S. 39], um so effektiv in gesunden Beziehungen und mit wenigen Konflikten [22, S. 40] die Lehre und das Lernen zu gestalten. Die Kommunikation findet nach Patrice Ras zwischen verantwortlichen, ebenbürtigen Erwachsenen auf Augenhöhe statt, indem Vorschläge gemacht, verhandelt, überzeugt oder Dinge in die Hand genommen werden [22, S. 45], um so langfristig effektiv das Lehren und Lernen zu gestalten.

Ein guter Lehrender vermittelt den Lernenden, auf die typischen geistig ineffektiven Neigungen zu achten: Kritisieren, Konkurrieren, Vergleichen oder Klagen (nach Anne van Stappen und Stephen R. Covey [25, S. 23-24], [10]). Ein guter Lehrender sollte den Lernenden vermitteln, sich selbst wahrzunehmen (Selbstwahrnehmung), Selbstempathie/Bewusstheit zu empfinden, sich gesund selbst zu behaupten (Selbstbehauptung/Mut; nach Patrice Ras: gesunde und klare Vorschläge machen, verhandeln, überzeugen und Dinge in die Hand nehmen [22, S. 45]) und Empathie/Verständnis für das Gegenüber zu empfinden (nach Anne van Stappen und Stephen R. Covey [25, S. 23-24][10]).

Es soll hier noch erwähnt sein, das Kritik an den Lehrenden oder Lernenden vergleichsweise eher ineffektiv ist, was die Verhaltensänderung angeht, sondern eher ein Erfolgserlebnis oder eine Verstärkung (siehe Corell [9, S. 189]) die gewünschte Verhaltensänderung bewirkt. Somit ist als Konsequenz anzuerkennen, das mehr Lob, Anerkennung und Ermutigung [26, S. 26-27] [7, S. 80-85] statt Tadel und Kritik [9, S. 162] die Effektivität der Lehre und der Lernens steigert, um so auch mutige und mit Selbstvertrauen [9, S. 161] ausgestattete Lernende zu erziehen.

\section{Lehren und Lernen durch Überprüfung im Unterricht}

Sofern der Lernstoff durch Chunks [7, S. 170-173] (kleinen Einheiten) vermittelt wurde, kann nach jedem Chunk durch einen Onlinefragebogen [13] das Ergebnis der Vermittlung und die Effektivität der Lehrmethode direkt überprüft werden. Wir konnten die Lehrmethode Onlinefragebogen im Rahmen der objektorientierten Programmierung in der Fernlehre mit den Pods in Adobe Connect verwenden [13] und dadurch Missverständnisse im OOP direkt ausräumen.

Hausarbeiten ermöglichen eine selbstständige, mehrseitige Anwendung und schriftliche Ausarbeitung des Gelernten. Die Sichtung und das Feedback zu den Hausarbeiten 
ermöglicht den Lernenden zu verstehen, ob die Lernziele im Unterricht zu einen Thema erreicht wurden (Feedback ist Teil des Lernprozesses nach Phil Races Ripple-Theorie [18, S. 168][13, 14]). Klausuren ermöglichen, den aktuellen Wissenstand und Kompetenzstand mittels verschiedener Aufgabenstellungen zu überprüfen. Wir denken dennoch, dass die Vermittlung und Überprüfung von prozeduralen Wissen und Faktenwissen innerhalb eines individuellen Praxisprojekts Vorrang vor der Überprüfung des größtenteils faktischen Wissens in einer Klausur haben sollte [2, 16], da eine Aktivierung des Lernenden, wie bereits erwähnt, effektiver ist [9, 14].

Kontrolle oder eine Überprüfung des Gelernten ist weniger häufig notwendig, wenn der Lernende motiviert ist [9, S. 158-162]. Wir haben festgestellt, dass insbesondere in Programmiertechniken, objektorientierter Programmierung [13] oder Datenbankentwicklung und Datenbankdesign [14] ein hohes Maß an Motivation und sehr gute Lernergebnisse zu erkennen waren, wenn ein Projekt oder Übungen vom Lernenden selbst gewählt und mit vorheriger Anleitung des Lehrenden ausgearbeitet werden konnten.

Die Lernleistungen können entweder summativ und formativ überprüft werden [16, S. 11]. Bei einer summativen Überprüfung wird eine Punktzahl, Note oder ein Prädikat angeboten [16, S. 11]. Die formative Überprüfung ermöglicht die Überprüfung des Lernfortschritt [16, S. 11] mit Feedback, bspw. bei Erreichung eines Lernziels innerhalb eines Praxisprojektes [14], welche sich nach unseren Erfahrungen als effektiv für das Lehren und Lernen in der Informatik und Wirtschaftsinformatik erwies (gemessen bspw. an der Zeit der Vermittlung oder erreichten Lernziele) [13, 14].

\section{Lehren und Lernen durch Gedächtnis im Unterricht}

Werner Corell definiert verschiedene Gesetze zum Gedächtnis [9, S. 183-187] um die Effektivität des Lehrens und des Lernens zu steigern:

- Gesetz der Kontiguität. Zwei Lerngegenstände, die zeitlich miteinander erfahren werden, werden erinnert. Zum Beispiel kann das Öffnen einer IDE mit der Erstellung einer Java-Datei erinnert werden.

- Gesetz der Sukzession. Zwei Lerngegenstände, die unmittelbar aufeinander folgen, werden erinnert; bspw. kann das Erstellen einer Klasse und dessen Variablen nacheinander stattfinden.

- Gesetz der Ähnlichkeit. Wenn sich zwei Lerngegenstände ähnlich sind, so werden sie gemeinsam erinnert. Zum Beispiel sollte die Verwendung eines Texteditors nicht direkt nach der Verwendung einer IDE gelernt werden, weil sich die Erinnerungen vermischen und nicht mehr exakt erinnert werden könnten.
- Gesetz des Kontrastes. Zwei Lerngegenstände, die einander unähnlich sind, können miteinander abgerufen werden. Die Verwendung einer IDE kann mit der Verwendung von Softwaremustern [5] gelernt werden.

Hemmings et al. [18, S. 31] unterscheidet zwischen folgenden Gedächtnisformen:

- Episodisches Gedächtnis. Es werden Erfahrungen bspw. bei der Bewältigung eines Praxisprojektes [14] abgerufen. Durch die stetige Interaktion mit dem Lehrobjekt konnte der Lerngegestand mit sensorischen und emotionalen Informationen verknüpft werden.

- Semantisches Gedächtnis. Es können bspw. faktische Informationen wie CPU-, RAM-Größen in einem Computer vom Lernenden gespeichert und abgerufen werden.

- Arbeitsgedächtnis. Es können im Laufe der Programmierung fünf bis sieben Dinge (bspw. Variablenzustände) gleichzeitig im Arbeitsgedächtnis gehalten werden, um das Programmierproblem zu lösen.

- Prozeduralgedächtnis. Erlernte Aktionen, wie zum Beispiel das Definieren einer Klasse, können ohne bewusstes Abrufen erstellt werden.

- Implizitgedächtnis. Es werden Fehlerquellen, unbewusste Erinnerungen zum Programmieren abgerufen, um den Fehler (Bug oder Issue [12]) zu vermeiden.

\section{Lehren und Lernen durch Aufmerksamkeit im Unterricht}

Corell unterscheidet in objektive und subjektive Formen der Aufmerksamkeit [9, S. 150-158] um die Effektivität des Lehrens und Lernens zu steigern. Objektive Formen der Aufmerksamkeit sind [9, S. 150-153]:

- Bewegung und Veränderung. Abwechslungsreiche Gestik und Mimik und Veränderung der Tonlage und Lautstärke bewirken mehr Aufmerksamkeit im Unterricht, zum Beispiel beim Beginn der Vorlesung.

- Neuheit oder Einmaligkeit. Wenn etwas neu und einmalig ist, wird mehr Aufmerksamkeit darauf gelegt, zum Beispiel bei Inhalten in der Informatik zu einer neuen Technologie oder einem neuen Thema.

- Größe. Die Größe der Objekte beeinflusst die Aufmerksamkeit, welche auch die Wirksamkeit der Objektes beeinflusst. Es ist ein Poster oder Tafelbild denkbar, das für den Unterricht oder Inhalte unterstützend verwendet wird.

- Intensität eines Reizes. Farben und Geräusche führen zur erhöhter Aufmerksamkeit. Wichtige Stellen im Unterrichtsmaterial können bspw. farbig ausgestaltet werden. Der Unterricht kann durch ein Geräusch begonnen werden. 
- Dauer bzw. Wiederholung. Einem Lerngegenstand wird wiederholt Aufmerksamkeit geschenkt, wenn auch nur bis zu einem gewissen Grad. Sodann tritt eine Gewöhnung ein. Zu häufige Wiederholungen im Unterricht sollten vermieden werden.

- Der Faktor der Farbe. Eine farbige Gestaltung des Materials bewirkt mehr Aufmerksamkeit.

Subjektive Formen der Aufmerksamkeit sind [9, S. 153158]:

- Der Zweck oder die Einstellung des Augenblicks. Der Zweck des Lerngegenstands wie das Lernen von Theorien für das Lösen eine praktischen Problems in der Informatik erhöht die Aufmerksamkeit für das Lernen des Lerngegenstands.

- Das Pflichtbewusstsein. Das Pflichtbewusstsein zu lernen, um die Prüfungen sehr gut zu bestehen und seine berufliche Pflicht als verantwortungsvoller und kompetenter Informatiker wahrzunehmen, erhöht die Aufmerksamkeit für den Lerngegenstand.

- Erziehung oder Gewöhnung. Eine Anknüpfung an die Erfahrungen des Lernenden sorgt für eine erhöhte Aufmerksamkeit für den Lerngegenstand. Zum Beispiel kann ein Praxisprojekt zu einem Lernobjekt für den Lernenden bereitgestellt werden, welches die Lernenden bereits kennen, wie zum Beispiel die Ausgestaltung einer Datenbank für eine Verkaufsseite von Amazon.com [14].

- Anknüpfung an Triebtendenzen. Die Gestaltung von Lehrgruppen in informellen Gruppen, in denen beide Geschlechter zusammenarbeiten, kann zur erhöhten Aufmerksamkeit bei den Lernenden führen. Die Möglichkeit zur Erlangung von mehr Prestige, Leistung, Überlegenheit und Macht kann zu erhöhter Aufmerksamkeit und effektiverem Lehren und Lernen führen [9, S. 158].

Lernende sind aufmerksamer bei der Bewältigung einer Aufgabe, wenn eine Belohnung erwartet wird [6, S. 130]. Dies kann beispielsweise durch zu erwartendes Lob und zu erwartende Anerkennung erfolgen [9, S. 162].

Sicherlich ist noch zu erwähnen, dass ein Konsum von Kaffee und Koffein (50-300 mg) auch Aufmerksamkeit, Energie und Konzentration fördert [1, S. 155].

\section{Lehren und Lernen mit Beachtung einer Lernstörung im Unterricht}

Wir denken, dass diverse Lernstörungen (bspw. hat jeder fünfte Dyslexie [8, S. 131]) bereits zu erheblichen Schwierigkeiten beim Lernen von Informatik und der Tätigkeit des Programmierens führen können. Carter et al. [6, S. 248] unterscheiden u. a. drei Lernstörungen:
- Dyslexie. Eine stark eingeschränkte oder bereits schwache Lese- und Schreibfähigkeit kann zu Programmierfehlern und Fehlererkennung beim Lernen von Programmiermustern oder Schreiben von Programmen führen. Die mögliche Unordnung der Gedanken kann zu ineffektivem Lernen führen. Lehrkonzepte, welche speziell auf die Fehlerkorrektur hinführen und trainieren, können das Lehren und Lernen von Programmierwissen effektiv machen und womöglich die Störung ausgleichen.

- Dyskalkulie. Die Schwierigkeit, mathematische Konzepte zu erfassen und Logiken sowie Rechnungen auszuführen, Zahlen oder Programmteile zu organisieren, kann die Effektivität des Lernens oder Lehrens von bspw. Programmieren gefährden. Durch die Vereinfachung von mathematischen Konzepten und Programmierregeln und durch das in sinnvollen Einheiten und schrittweise Vermitteln dieser Konzepte kann dieser Lernstörung begegnet werden und die Effektivität des Lehrens und Lernens erhöht werden.

- Spezifische Sprachentwicklungsstörung. Durch das mangelnde Verstehen von gesprochener Sprache kann dem Lehrenden nicht gefolgt werden, was das Lehren und Lernen ineffektiv macht. Videos $\mathrm{zu}$ den Lehrinhalten können helfen, auch dieser Lernstörung effektiv zu begegnen [14].

Der Großteil der Lernenden in den Fächern Informatik, Wirtschaftsinformatik und verwandten Fachgebieten sind Männer. Simon Baron-Cohen [7, S. 298-299], [3] unterscheidet zwischen dem männlichen Gehirn, das von seiner Struktur für das Verständnis und Aufbau von Systemen ausgerichtet ist, und dem weiblichen Gehirn, welches dagegen eher auf Emphatie ausgerichtet ist. Männer und Frauen haben ein ausgeglichenes Gehirn [7, S. 298]. Eine extreme Variante des männlichen Gehirns ist wie besessen von Systemen, was Simon Baron-Cohen als ein Gehirn mit Autismus beschreibt [7, S. 299]. Collin et al. [8, S. 217] charakterisieren u. a. ein autistisches Gehirn mit Defiziten in der sozialen Kommunikation und fixierten Interessen, was das gemeinschaftliche Lernen sowie das fächerübergreifende Erlernen von Themen zur Informatik, Wirtschaftsinformatik und verwandten Fachgebieten erschwert und ineffektiv macht.

\section{Einschränkungen zum Lehrbaummodell für effektives Lehren und Lernen}

Das Lehrbaummodell zielt darauf ab, Frustrationen zu vermeiden und den Unterricht effektiver zu gestalten. Es sei anzumerken, dass der Lehrende und die Lernenden sich stets um eine hohe Frustrationstoleranz, insbesondere bei der aktivierenden Unterrichtsform, bemühen sollten [9, S. 433-434]. Insbesondere wenn die durchgeführte Lehre 
Lernende aktiviert und verschiedene Meinungen im Raum diskutiert werden, so sollte sich der Lehrende und die Lernenden in stoischer Gelassenheit üben [9, S. 434], um die Lehre effektiv zu gestalten, sodass für alle der beste Konsens gefunden werden kann.

Ellis et al. [11] diskutieren verschiedene effektive Lehrund Lernmethoden, ohne ein Gesamtlehrmodell vorzuschlagen. Das Lehrbaummodell fokussiert auf Planung, Motivation und Überprüfung und weiterer Aspekte zur Reduzierung von Frustration und Erhöhung der Effektivität der Lehr- und Lernmethoden. Valentin Razmov diskutiert weitere effektive pädagogische Prinzipien, welche in Projekten in der Lehre von Software Engineering genutzt werden können [23]. Garg et al. [17] sagen, dass kontextsensitive Vermittlung von Wissen anhand von praktischen Fällen und mittels eines Lernzyklus (Case-Oriented Learning Environment [COSEEd]) zu mehr Lehr- und Lernerfolg und bspw. zur Verbesserung der Kommunikationskompetenz führen kann. Unser Lehrbaummodell behandelt u.a. auch den Aspekt Kommunikation, welcher für das Lehren und Lernen in der Informatik, Wirtschaftsinformatik und verwandten Fachgebieten effektiv ist.

\section{Zusammenfassung und Ausblick}

Wir konnten durch die Analyse zur Umsetzung effektiver Lehre in der Informatik, Wirtschaftsinformatik und verwandten Fachgebiete mittels unseres Lehrbaummodells aufzeigen, dass im Vergleich zum traditionellen Lehren und Lernen weitere Kompetenzen vom Lehrenden und auch vom Lernenden gefordert werden. Die Aktivierung während des Unterrichts, der freie Meinungsaustausch sowie das Lehren und Lernen auf Augenhöhe ermöglicht eine gemeinsame, effektive Gestaltung der Lehre und des Lernens. Zeitliche und energetische Ressourcen können durch die Aktivierung vom Lehrenden und Lernenden gespart werden. Eine effektive Planung sowie Motivierung des Unterrichts ermöglicht es, die Lernenden zu begeistern und vielleicht vorerst sekundär motivierte Lernende in primär motivierte Lernende zu wandeln, womit weniger Lernzielkontrollen notwendig sind und effektiv in der Informatik, Wirtschaftsinformatik und verwandten Fachgebieten gelehrt und gelernt werden kann.

Danksagung Wir danken der DIPLOMA Hochschule für die Unterstützung zur Veröffentlichung dieses Artikels und die Möglichkeit, in Wirtschaftsinformatik und Wirtschaftsingenieurswesen seit 2018 zu lehren. Wir danken dem Fachbereich Informatik der Universität Hamburg für die Möglichkeit, in der Lehre und in der Planung in der Informatik von 2013-2017 zu unterrichten. Wir danken der heutigen Ostbayerischen Technischen Hochschule Regensburg für die Lehraufträge in der Informatik und Elektro- und Informationstechnik. Wir danken den privaten Hochschulen der Stadt Hamburg für die Möglichkeit, in der Informatik, insbesondere Fachinformatik, zu lehren und zu lernen. Ohne die langjährige Erfahrung und Auseinandersetzung mit Lehr- und Lernmodellen und ihren Aspekten in der Informatik, Wirtschaftsinformatik und verwandten Fachgebieten wäre diese Veröffentlichung nicht möglich geworden.

Funding Open Access funding enabled and organized by Projekt DEAL.

Open Access Dieser Artikel wird unter der Creative Commons Namensnennung 4.0 International Lizenz veröffentlicht, welche die Nutzung, Vervielfältigung, Bearbeitung, Verbreitung und Wiedergabe in jeglichem Medium und Format erlaubt, sofern Sie den/die ursprünglichen Autor(en) und die Quelle ordnungsgemäß nennen, einen Link zur Creative Commons Lizenz beifügen und angeben, ob Änderungen vorgenommen wurden.

Die in diesem Artikel enthaltenen Bilder und sonstiges Drittmaterial unterliegen ebenfalls der genannten Creative Commons Lizenz, sofern sich aus der Abbildungslegende nichts anderes ergibt. Sofern das betreffende Material nicht unter der genannten Creative Commons Lizenz steht und die betreffende Handlung nicht nach gesetzlichen Vorschriften erlaubt ist, ist für die oben aufgeführten Weiterverwendungen des Materials die Einwilligung des jeweiligen Rechteinhabers einzuholen.

Weitere Details zur Lizenz entnehmen Sie bitte der Lizenzinformation auf http://creativecommons.org/licenses/by/4.0/deed.de.

\section{Literatur}

1. \#dkinfografik. Was wir essen - Alles über unsere Nahrung (2018) (Lektorat Gritschneder Kathrin, Übers. Krabbe W). Dorling Kindersley, München

2. Anderson L, Krathwohl D (2001) A taxonomy for learning, teaching and assessing. Longman, New York

3. Baron-Cohen S (2004) Vom ersten Tag an anders. Walter, Düsseldorf - Zürich

4. Ruhr-Universität Bochum Lernzieltaxonomien im Vergleich. https:// dbs-lin.ruhr-uni-bochum.de/lehreladen/planung-durchfuehrungkompetenzorientierter-lehre/lehr-und-lernziele/lernzieltaxonomienim-vergleich/. Zugegriffen: 10. Aug. 2021

5. Bruegge B, Dutoit AH (2013) Object-oriented software engineering-using uml, patterns, and java

6. Carter R, Aldridge S, Page M, Parker S (2019) Das Gehirn - Anatomie, Sinneswahrnehmung, Gedächtnis, Bewusstsein, Störungen. Dorling Kindersley, München

7. Collin C, Benson N, Ginsburg J, Grand V, Lazyan M, Weeks M (2012) Big Ideas. Das Psychologie-Buch. Dorling Kindersley, München

8. Collin C, Jackson T, Parker S, Smith G, Collin T, Drew L, Horobin W, John K, Yhnell E, Temple N, Watt S (2020) \#dkinfografik. Das menschliche Gehirn und wie es funktioniert. Dorling Kindersley, München

9. Correll W (2015) Menschen durchschauen und richtig behandeln: Psychologie für Beruf und Familie. mvg, München

10. Covey SR (2018) Die 7 Wege zur Effektivität: Prinzipien für persönlichen und beruflichen Erfolg. GABAL, Offenbach

11. Ellis HJ, Demurjian SA, Naveda JF (2008) Software engineering: effective teaching and learning approaches and practices. IGI Global, Hershey

12. Ellmann M (2018) Natural language processing (nlp) applied on issue trackers. In: Proceedings of the 4th ACM SIGSOFT International Workshop on NLP for Software Engineering, Association for Computing Machinery. Association for Computing Machinery, New York, NY, USA, S 38-41 https://doi.org/10.1145/3283812. 3283825 
13. Ellmann M (2021) Fernlehren und fernlernen von objektorientierter programmierung (oop). Informatik Spektrum 44(2):115-121

14. Ellmann M (2021) Umschulung zum IHK-Fachinformatiker in Anwendungsentwicklung und Systemintegration. Informatik Spektrum 44:328-338. https://doi.org/10.1007/s00287-021-01371-x

15. Ellmann M, Oeser A, Fucci D, Maalej W (2017) Find, understand, and extend development screencasts on youtube. In: Proceedings of the 3rd ACM SIGSOFT International Workshop on Software Analytics, Association for Computing Machinery. Association for Computing Machinery, New York, NY, USA, S 1-7 https://doi.org/ $10.1145 / 3121257.3121260$

16. Universität Zürich Bereich Lehre Arbeitsstelle für Hochschuldidaktik (2010) Taxonomie-Matrix zur Analyse und Selbstevaluation von Hochschullehre (TAMAS). Dossier Unididaktik, Bd. 1/10. Universität Zürich Bereich Lehre - Arbeitsstelle für Hochschuldidaktik,

17. Garg K, Sureka A, Varma V (2015) A case study on teaching software engineering concepts using a case-based learning environment. In: Lichter H, Anwar T, Sunetnanta T, Vianden M, Dubey A, Celis LE, Grant ES, Shankararaman V (Hrsg) Joint Proceedings of the 3rd International Workshop on QuASoQ, WAWSE and the 1st International Workshop on CMCE co-located with APSEC 2015 New Delhi, India, 1. Dez. 2015. Bd. 1519, S 71-78 (CEURWS.org, CEUR Workshop)

18. Hemmings J, Collin C, Ganz JG, Lazyan M, Black A (2019) \#dkinfografik. Psychologie im Alltag - Wie wir denken, fühlen und handeln. Dorling Kindersley, München
19. Lalley J, Miller R (2007) The learning pyramid: Does it point teachers in the right direction. Education 128(1):16

20. Levine R (2015) Die große Verführung. Piper, München

21. Petitcollin C (2016) Psychospiele durchschauen und die eigene Rolle verändern. TRINITY, München

22. Ras P (2015) Konflikte meistern und harmonischere Beziehungen führen. TRINITY, München

23. Razmov V (2007) Effective pedagogical principles and practices in teaching software engineering through projects. 2007 37th Annual Frontiers In Education Conference - Global Engineering: Knowledge Without Borders, Opportunities Without Passports, S S4E-21-S4E-26 https://doi.org/10.1109/FIE.2007.4418158

24. Taubner D (2021) Gründung und Entwicklung von IT-Häusern. Informatik Spektrum 44:387-388. https://doi.org/10.1007/s00287021-01400-9

25. Stappen A van (2015) Das kleine Übungsheft - Grenzen setzen, nein sagen, Bd 5. Trinity, München

26. Weeks M (2019) Kernfragen. Psychologie. Dorling Kindersley, München

Hinweis des Verlags Der Verlag bleibt in Hinblick auf geografische Zuordnungen und Gebietsbezeichnungen in veröffentlichten Karten und Institutsadressen neutral. 